\title{
El tratamiento del cáncer de próstata: Presente y futuro, realidades y posibilidades
}

\author{
Vera Donoso CD. \\ Servicio de Urología. Hospital Universitario La Fe. Valencia.
}

Actas Urol Esp. 2007;31(6):575-579

\section{RESUMEN}

\section{EL TRATAMIENTO DEL CÁNCER DE PRÓSTATA: PRESENTE Y FUTURO, REALIDADES Y POSIBILIDADES}

La determinación universal del PSA y su consecuencia más importante, la detección del cáncer de próstata en estadio localizado, ha originado una migración rotundamente favorable del estadio tumoral al momento del diagnóstico. Ello implica un incremento exponencial de la necesidad de tratamientos primarios con intención curativa. La prostatectomía radical con preservación de las bandeletas neurovasculares es la "regla de oro" para tratar el cáncer de próstata localizado. En el último quinquenio, la cirugía laparoscópica y la cirugía robótica se han incorporado de lleno, como refinadas variantes terapéuticas. Estos abordajes terapéuticos y las técnicas nuevas y emergentes, aquellas intervenciones que no constituyen "práctica rutinaria" en el tratamiento del cáncer de próstata, guiadas por imágenes presenta una actividad investigadora constante. Efectuamos aquí una revisión de todas las posibilidades del tratamiento del cáncer prostático.

Palabras clave: Próstata. Cáncer. Tratamiento. Nuevas tecnologías.

\section{ABSTRACT \\ THE TREATMENT OF PROSTATIC CANCER: PRESENT AND FUTURE, REALITIES AND POSSIBILITIES}

The routine determination of PSA has generated an important consequence: a really favourable migration of the tumour stage at the moment of diagnosis. It implies an exponential increase in the requirement of primary treatments with curative intention. Radical prostatectomy with preservation of neuro-vascular bundles is the gold standard for the treatment of localized prostate cancer but laparoscopic and robotic surgery have arrived with force during last five years. These therapeutic approaches and the new emerging techniques (those interventions that are not routine practice in the prostate cancer) guided by images present a constant research activity. Here, we perform a review of every possibility to treat the cancer of prostate.

Keywords: Prostate. Cancer. Treatment. New technology.

$\mathrm{E}^{1}$ cáncer de próstata es el segundo tumor maligno más frecuente entre los hombres y representa el 11,7 \% de todos los tumores masculinos. Se estima que cada año se diagnostican, a nivel mundial, 680.000 nuevos casos. En los países desarrollados, su frecuencia ha aumenta- do en la última década con un ritmo alto, convirtiéndose en ciertas áreas, en el tumor masculino más común.

Se ha situado en el tercer lugar de la lista de causas de muerte en los hombres $(22,9$ defunciones por 100.000 habitantes en el año 2000) en el 
territorio español, después del cáncer de pulmón y colo-rectal. Antes de los cincuenta años de edad, su aparición es infrecuente, pero, a partir de entonces su incidencia aumenta de modo persistente y más rápido que en cualquier otro tumor. Los hombres mayores de 65 años, aquellos con menor expectativa de vida, son el subgrupo de población sobre el que se produce el impacto mayor de este crecimiento (90\%). Según el informe Situación del Cáncer en España ${ }^{1}$ la tasa ajustada de incidencia estimada en 1998 era de 45 casos por 100.000 habitantes, encontrándose entre las más bajas de Europa, junto con las de Italia y Grecia. ¿Es esto real? Es muy probable que no. Sousa Escandón et al. ${ }^{2}$, recientemente, revelan que, en Galicia, la incidencia en un estudio muy controlado estaría en 143 casos por 100.000 varones y Herranz et al. ${ }^{3}$ ya advirtieron de una tasa de 100,4 casos/100.000 varones en la Comunidad de Madrid en el año 2000. En cuanto a la supervivencia, se considera que en nuestro país, la supervivencia global es de $65,5 \%$ a los 5 años del diagnóstico (casos diagnosticados entre 1990 y 1994). Este aspecto ha mejorado sensiblemente porque en el quinquenio de 1980 a 1985, la supervivencia era de $48 \%$. Indudablemente, la detección precoz mediante el uso generalizado del PSA ha permitido detectar tumores pequeños que se tratan con un pronóstico excelente.

En nuestro país, cada año mueren 5.900 hombres por causa de este tumor constituyendo el $10 \%$ de la mortalidad masculina por cáncer y el $2,8 \%$ de la mortalidad masculina general. La edad media de los pacientes que mueren en España por cáncer de próstata es de 75 años.

\section{Tratamiento del Cáncer de Próstata Localizado}

El tratamiento estándar para el cáncer de próstata localizado se reduce, actualmente, a tres realidades:

- Prostatectomía radical.

- Radioterapia externa o intersticial.

- Seguimiento expectante.

Los dos primeros originan una tasa no despreciable de efectos deletéreos, los más frecuentes, impotencia e incontinencia urinaria, aunque los refinamientos técnicos han mejorado la eficacia y la seguridad. El tercero es una modalidad de tratamiento para aplicar a casos muy seleccionados y con vigilancia muy estricta.

Esta situación ha originado una pujante carrera por desarrollar diferentes formas de tratamiento que alcancen la máxima eficacia oncológica, tengan la menor tasa posible de efectos secundarios y sean escasamente agresivos. Entre ellos destacamos los siguientes:

- Prostatectomía Radical mediante Minilaparotomía.

- Prostatectomía Radical Laparoscópica.

- Prostatectomía Radical Robótica.

- Braquiterapia.

- Ultrasonido de Alta Frecuencia (HIFU).

- Terapia térmica con nanopartículas magnéticas.

- Radioterapia conformada de intensidad modulada (IMRT).

- Crioterapia.

- Ablación Intersticial Transperineal con Radiofrecuencia.

- Terapia Fotodinámica.

Todos estos abordajes los vamos a analizar en este número monográfico a través de una serie de revisiones con el soporte de la propia experiencia de varios grupos nacionales y extranjeros, y en los métodos cuya implantación generalizada aún está esperando la sanción del tiempo y el volumen de pacientes, generosas exposiciones que nos ayudan a entrever un futuro que se acerca a grandes pasos.

Dentro del apartado quirúrgico que considera la Prostatectomía Radical como regla de oro, el Dr. Veiga, del Servicio de Urología del Complejo Hospitalario Juan Canalejo de La Coruña, plantea su experiencia con la Minilaparotomía, que puede ser útil para muchos grupos de trabajo que, por diversas razones, han descartado las técnicas laparóscopicas.

La Prostatectomía Radical Laparoscópica Extraperitoneal (EERPE) fue analizada para este número Monográfico, de modo magistral y con un soporte de experiencia muy importante por el Profesor Jens Uwe Stolzenburg, del Departamento de Urología de la Universidad de Leipzig (Alemania), pero razones de fuerza mayor, hicieron adelantar su publicación al número de Junio (2006) de nuestra revista. Remitimos, por lo tanto, a esta cita, a los interesados en este abordaje. 
$\mathrm{El}$ auge de la cirugía robótica se refleja de modo innegable en tres artículos que nos exponen la amplia experiencia del Prof. Dr. Hubert John del Departamento de Urología del Hospital de Zurich y la Clínica Hirslanden (Suiza) con su técnica de Prostatectomía Radical Robótica Extraperitoneal (más de 300 casos, que lo convierten en un referente europeo de la urología robótica) y la labor pionera que la Fundación Puigvert, con el liderazgo del Dr. Humberto Villavicencio, ha desarrollado en esta tecnología urológica en nuestro país que, además, demuestra las posibilidades de conversión de un cirujano experto a la cirugía robótica sin mayores dificultades. Cierra este apartado, la reflexión que realizan los Dres. Martines Salamanca (Department of Urology Weill Medical College at Cornell University, New York, USA) y Antonio Allona (Equipo de Urología de La Clínica Ruber Internacional), acerca de los fundamentos para que este sistema quirúrgico, sofisticado y costoso, tenga un sitio dentro de la sanidad pública española.

La radioterapia externa no se ha detenido en el abordaje triconformacional que ha disminuido de modo importante la morbilidad de esta forma de tratamiento. El Dr. Livi, del Departamento de Oncología Radioterápica de la Universidad de Florencia nos avanza sus resultados, y deja ver que es posible incrementar las dosis administradas mediante la Radioterapia de Intensidad Modulada (IMR) sin aumentar los efectos secundarios, especialmente, los que conciernen al aparato digestivo.

La Braquiterapia como la última variante, relativamente novedosa de los tratamientos radioterápicos, es analizada por el equipo del Hospital Universitario Central de Asturias, que une a urólogos y radioterapeutas, encabezados por el Dr. Ramón Abascal, en el trabajo diario, y que, en España, abanderan esta técnica con una casuística muy amplia (800 pacientes) que les permite solvencia y rigor en su exposición.

Las posibilidades que nos brindan los Ultrasonidos de Alta Frecuencia (HIFU) son revisadas, exhaustiva y eficazmente, por el Servicio de Urología del Hospital Gregorio Marañón (Madrid). Se observa una implantación creciente de centros que utilizan con asiduidad esta técnica y el Dr. Lledó y colaboradores nos ofrecen una visión realista e independiente.
El Profesor Truls E. Bjerklund Johansen, del Servicio de Urología del Hospital Telemark de Porsgrunn, Noruega, pasa revista, diligentemente, a la Criocirugía como tratamiento primario en el cáncer de próstata, especialmente de la población escandinava, cuando empiezan a aparecer experiencias en este campo, en territorio español, más orientados al rescate de pacientes con un fracaso terapéutico previo que al tratamiento primario.

La Ablación mediante Radiofrecuencia Intersticial (RITA) en el tratamiento del cáncer de próstata órgano-confinado es revisada por el Dr. Bonillo, de nuestro Servicio de Urología. Se ha mostrado como una alternativa terapéutica segura y eficaz en el tratamiento del cáncer de próstata órgano-confinado, con escasas complicaciones, con escaso tiempo de ablación (alrededor de 10 minutos), con posibilidad de controlar el tamaño de las lesiones inducidas ${ }^{6,8}$. Tiene la capacidad de ser una técnica fácilmente reproducible por cualquier urólogo y la posibilidad de repetir el tratamiento si es preciso.

El Dr. Manfred Johannsen y sus colegas, del Departamento de Urología de la Universidad Charité de Berlín, nos introducen en su novedosa técnica para terapia térmica intersticial mediante la inyección de un fluido que contiene nanoparticulas superparamagnéticas biocompatibles (fluido magnético) que se somete luego a campos magnéticos alternantes y es la primera técnica térmica que, utilizando nanopartículas magnéticas, ha sido admitida para la realización de ensayos clínicos.

El Dr. Ramírez Backhaus, de nuestro grupo evalúa la Terapia Fotodinámica, una técnica de futuro prometedor que se basa en la aplicación de una fuente de energía en forma de luz con una determinada longitud de onda, sobre un tejido previamente fotosensibilizado mediante un compuesto químico, en presencia de oxígeno. De este modo se generan gran cantidad de radicales libres y derivados del oxígeno (compuestos hidroxilo) que producen necrosis del tejido. Actualmente se está estudiando la terapia del blanco vascular con el fármaco fotosensibilizante "palladium bacteriophephorbide" TOOKAD ${ }^{\circledR}$. El fármaco se activa en el torrente vascular y daña la estructura vascular del tumor con la consecuente necrosis tumoral. Varios ensayos clínicos se 
encuentran en marcha utilizándola en pacientes con cáncer de próstata localizado como tratamiento primario o de rescate.

Conclusiones: ¿Qué esperamos de las técnicas de tratamiento primario del cáncer de próstata órgano confinado?

La determinación universal del PSA y su consecuencia más importante, la detección del cáncer de próstata en estadio localizado, ha originado una migración rotundamente favorable del estadio tumoral al momento del diagnóstico. Ello implica un incremento exponencial de la necesidad de tratamientos primarios con intención curativa. La prostatectomía radical con preservación de las bandeletas neurovasculares descrita por Patrick Walsh y colaboradores, en la década de los 80 , se ha convertido en la "regla de oro" para tratar el cáncer de próstata localizado. En el último quinquenio, la cirugía laparoscópica y la cirugía robótica se han incorporado de lleno, como refinadas variantes terapéuticas.

Las técnicas nuevas y emergentes, aquellas intervenciones que no constituyen "práctica rutinaria" en el tratamiento del cáncer de próstata, (como la cirugía convencional, la radioterapia convencional, seguimiento activo) y que generan discusión y evaluación en la literatura médica, guiadas por imágenes presentan una actividad investigadora constante. Muchos interrogantes que devienen en metas nos plantean el futuro:

- ¿Cómo podemos evitar la alta tasa de disfunción eréctil que todas las técnicas generan?

- Mejorando la detección con imágenes ¿Podremos llegar al tratamiento eficaz que afecte sólo al área tumoral, sin extirpar ni tratar todo la próstata, a modo de una lumpectomía?

- Estas técnicas no invasivas, ¿conseguirán anular la tasa de incontinencia urinaria, el efecto adverso que más afecta la calidad de vida de nuestros enfermos?

- Con esta finalidad, deben implementarse los siguientes objetivos:

1) estandarizar las definiciones de eventos adversos,

2) crear ensayos clínicos aleatorizados con un seguimiento suficientemente largo (en términos de supervivencia global) y evaluación de la calidad de vida.
- Los estudios de calidad de vida deben incluir pacientes en tratamiento, pacientes en observación y sujetos sanos.

\section{Tratamiento del Cáncer de Próstata Localmente Avanzado}

Debido a la variabilidad en las vías de diseminación linfática pélvica desde la glándula prostática, la identificación del primer ganglio de una posible diseminación tumoral en un paciente dado, sería la solución ideal para el compromiso quirúrgico que implica una prostatectomía radical que pretende ser curativa.

El Profesor Janetschek y su equipo del Departamento de Urología del Hospital Elisabethinen de Linz (Austria) nos introducen en el concepto de ganglio centinela en el cáncer de próstata mediante la linfadenectomía guiada por radioisótopos, que nos ha explicado muchos de los fracasos de la prostatectomía radical convencional y tiene un gran potencial para ofrecer mejores expectativas para los pacientes con enfermedad localmente avanzada.

\section{Tratamiento del Cáncer de Próstata Diseminado}

Pocos avances se han reportado en los últimos años para el tratamiento del cáncer de próstata avanzado localmente o a distancia. La irrupción del tratamiento con docetaxel que demuestra una actividad más patente, es poco apreciable por la comunidad urológica después de tantos años sin un papel claro y favorable de la quimioterapia sistémica. Nos aproximamos también al advenimiento de los tratamientos del futuro como los antagonistas de la endotelina, anticuerpos contra antígenos de membrana específicos de la próstata, vitamina $\mathrm{D}$, agentes antiangiogénicos, compuestos antisentido y los inhibidores de los receptores de los factores de crecimiento.

Los doctores Kaskel y Veelken, del Centro Universitario de Cáncer de Freiburgo (Alemania) diseccionan el tratamiento inmunológico del cáncer de próstata, particularmente, la terapia con vacunas de células dendriticas mediante la experiencia aportada por una cincuentena de ensayos (aproximadamente mil pacientes) que revelan la inocuidad de este abordaje, así como su transitoria eficacia. 
Por último, el Profesor Winston Tan (Clínica Mayo, Florida) hace una revisión exhaustiva de las nuevas opciones de tratamiento para el cáncer de próstata metastásico hormonorefractario. $\mathrm{El}$ docetaxel y su combinación con múltiples agentes de las más diversas familias (satraplatino, bevacizumab y sorafenib, calcitriol, imatinib, ixabepilone, atrasentan) están siendo evaluados con la intención de mejorar la supervivencia en estos pacientes.

\section{Agradecimientos}

Agradezco, personalmente y en nombre de ACTAS UROLOGICAS ESPAÑOLAS, el trabajo desinteresado que han hecho, este grupo de urólogos, oncólogos médicos y radioterapeutas, de origen tan diverso, al revisar estos temas que, sin duda, tendrán repercusión en la comunidad urológica.

\section{REFERENCIAS}

1. López-Abente G et al. La Situación del Cáncer en España. Ministerio de Sanidad y Consumo. Madrid 2005.

2. Sousa Escandón A, Sabell Salgues F y Miembros del Grupo de estudio Breogan 2003-2007 de la Sociedad Gallega de Urología. Referencia al artículo "Situación epidemiológica del cáncer de próstata en España”. Actas Urol Esp 2006; 30 (8):843.

3. Herranz F, Arias F, Arrizabalaga M, Calahorra FJ, Carballido $\mathrm{J}$ et al. El cáncer de próstata en la comunidad de Madrid en el año 2000: Incidencia. Actas Urol Esp 2003; 27(5): 323334 .

Correspondencia autor: Dr. César David Vera Donoso Servicio de Urología

Hospital Universitario La Fe

Avda. Campanar, 21 - 46009 Valencia

Tel.: 963862700

E-mail: cdvera@pulso.com

Información artículo: Original 